\section{Oncologia de Cabeça e Pescoço: enquadramento epidemiológico e clínico na avaliação da Qualidade de Vida Relacionada com a Saúde}

\section{Head and Neck Cancer: Health Related Quality of Life Assessment considering clinical and epidemiological perspectives}

\author{
Augusta Silveira ${ }^{1,1,1,11}$ \\ Joaquim Gonçalvesıv \\ Teresa Sequeirall,v \\ Cláudia Ribeiro ${ }^{\mathrm{VI}, \mathrm{VII}}$ \\ Carlos Lopes ${ }^{\text {I,VIII }}$ \\ Eurico Monteiro' ${ }^{1, \mathrm{VIII}}$ \\ Francisco Luís Pimente ${ }^{i x, x}$ \\ ' Instituto de Ciências Biomédicas Abel Salazar da Universidade do Porto. \\ "Universidade Fernando Pessoa. \\ III Centro de Estudos e Investigação em Saúde da Universidade de Coimbra. \\ Iv Intituto Politécnico do Cávado e Ave. \\ ${ }^{\vee}$ Instituto de Biologia Celular e Molecular \\ vi Universidade Católica Portuguesa \\ VII Universidade de Santiago de Compostela \\ VIII Instituto Português de Oncologia do Porto \\ Ix Universidade de Aveiro \\ ${ }^{x}$ Hospital Infante D. Pedro, Aveiro, Portugal
}

\section{Resumo}

A importância das doenças oncológicas como causa de morbilidade e mortalidade está em crescimento, sendo reconhecido o seu impacto social e peso global pelos custos económicos e sociais envolvidos na sua prevenção, tratamento e reabilitação. As patologias oncológicas de cabeça e pescoço representam um dos seis tumores malignos mais prevalentes em todo o mundo, com um valor estimado de 900.000 novos casos diagnosticados anualmente em escala mundial. Estes doentes oncológicos apresentam deterioração de funções básicas que, quando percepcionadas, têm impacto negativo na sua Qualidade de Vida. Um registo oncológico adequado permite uma análise mais rigorosa dos resultados obtidos na avaliação da Qualidade de Vida Relacionada com a Saúde. Este estudo incluiu 380 doentes oncológicos de cabeça e pescoço e demonstra que as mulheres apresentam resultados de Qualidade de Vida globalmente inferiores. Salienta-se ainda a importância do diagnóstico precoce em oncologia, que se relaciona frequentemente com melhores scores e conclui-se que a localização do tumor tem impacto sobre a autopercepção de Qualidade deVida. Os valores de Qualidade deVida Relacionada com a Saúde devem ser interpretados à luz das variáveis sociodemográficas e clínicas, para melhor se avaliar a Patologia Oncológica de Cabeça e Pescoço numa perspectiva epidemiológica no sentido de melhor compreender o processo saúde - doença.

Palavras-chave: Epidemiologia. Oncologia. Doentes Oncológicos de Cabeça e Pescoço. Qualidade de Vida. Patogênese. Prevenção.

Correspondência: Augusta Silveira. Universidade Fernando Pessoa - Faculdade de Ciências da Saúde. Rua Carlos da Maia, 296 | 4200-150 Porto, Portugal. e-mail: augusta@ufp.edu.pt 


\section{Abstract}

The importance of oncology diseases as a cause of morbidity and mortality is increasing worldwide, and their social impact is being recognized due to economic and social costs involved in prevention, treatment and rehabilitation. Head and neck cancer is one of the six most prevalent neoplasms worldwide, with an estimated 900,000 new cases diagnosed annually. Regardless of tumor site, deterioration of basic functions affecting head and neck areas are perceived and affect patients' lives. Appropriate cancer registration may provide a better analysis of health-related quality of life outcomes. In this study, 380 head and neck cancer patients were evaluated. The study showed that women have lower overall Quality of Life results. It also emphasizes the importance of early diagnosis, which often relates to stages with better prognosis and better Quality of Life outcomes. The study concluded that tumor location has an impact on Quality of Life self-perception. Values of Health Related Quality of Life should be analyzed along with socio-demographic and clinical variables in order to better understand the epidemiology, pathogenesis, and prevention of Head and Neck Cancer.

Keywords: Epidemiology. Oncology. Head and neck cancer patients. Quality of life. Pathogenesis. Prevention.

\section{Introdução}

\section{Patologia Oncológica de cabeça e pescoço: Epidemiologia}

A patologia oncológica representa a segunda causa de morte em Portugal. A sua importância como causa de morbilidade e mortalidade está em crescimento, e é reconhecido o seu impacto social ${ }^{1,2}$. O peso global das doenças oncológicas, pelos custos econômicos e sociais envolvidos na sua prevenção, tratamento e reabilitação tem vindo a aumentar ${ }^{1-4}$.

A epidemiologia é parte integrante da investigação oncológica. Dos estudos epidemiológicos emergem padrões que orientam as bases científicas e fundamentam a prática dos cuidados de saúde ${ }^{6-8}$.

No geral, a taxa de mortalidade por tumores malignos em Portugal é baixa comparada com a média da União Europeia ${ }^{2}$. No entanto, nos tumores malignos da cavidade oral, subjacente aos quais está um sinergismo entre o álcool e o tabaco, o risco de morrer em Portugal é maior do que na média da Comunidade Europeia ${ }^{9}$.

Os tumores malignos de cabeça e pescoço afetam preferencialmente os homens (o ratio homens/mulheres é de 2 - 5:1, dependendo da localização $)^{9}$. Sabe-se que os tumores malignos mais comuns na área de cabeça e pescoço são os da cavidade oral, representando cerca de 390.000 novos casos em escala mundial, sendo o $11^{\circ}$ tumor maligno mais comum no mundo e representando cerca de $5 \%$ de todos os tumores malignos ${ }^{10}$. Os tumores malignos da laringe representam $2 \%$ do número total de tumores malignos, com 160.000 novos casos por ano, que representam $0,6 \%$ do número total de tumores malignos. A proporção de tumores malignos que resultam de lesões pré-malignas varia entre 30 - $80 \%$. Este risco é maior nas mulheres e é dependente do tipo e da localização da lesão e da presença de lesões displásicas ${ }^{4,5}$.

Os tumores malignos da cabeça e pescoço contribuem para $4 \%$ das mortes no sexo masculino, representando a quinta causa 
de morte neste sexo por doença oncológica em Portugal. Nas mulheres, estes tumores são responsáveis por $1 \%$ da mortalidade por doenças oncológicas, a qual aparentemente não tem registado aumento ${ }^{9}$.

O prognóstico dos doentes com tumores malignos da cabeça e pescoço é condicionado pela localização e tamanho do tumor primário, com o envolvimento de gânglios regionais e com a idade. Além disso, alguns autores referem que a gradação do tumor é um factor de prognóstico particularmente relevante neste contexto ${ }^{3,10,11}$.

As taxas de sobrevivência aos 5 anos são inferiores a $50 \%$, geralmente favorável às mulheres ${ }^{3,10-12}$. As novas formas de tratamento não têm afetado de forma significativa as taxas de sobrevivência nos últimos 40 anos $^{13}$.

\section{Patologia Oncológica de cabeça e pescoço: Otimização da Qualidade de Vida}

Até 1960 não constavam dos planos curriculares das Faculdades de Medicina temas como: comunicação com o paciente; considerações psicossociais nas relações com os doentes e prevenção de doenças por mudança comportamental ${ }^{14}$.Todavia, áreas envolventes da Medicina têm sido paulatinamente integradas na prática médica contemporânea, complementando os modelos tradicionais de atuação numa perspectiva centrada no doente ${ }^{15-17}$. Nesta óptica, a avaliação da Qualidade de Vida (QdVRS) e sua implementação na prática clínica, constituem um método de recolhimento de um dos resultados de saúde reportados pelo doente mais usados em Medicina ${ }^{18-21}$.

A avaliação por rotina da QdVRS em Oncologia permite a obtenção de resultados clínicos que podem suportar as decisões clínicas, identificar necessidades desconhecidas e suportar um "stepped care model”; e a identificação de grupos de risco e providência de informação prognóstica. Estas contribuições são decisivas para se obter ganhos em saúde, desenvolver estratégias que optimizem a QdVRS e refinar os Sistemas de Saúde ${ }^{1,22-26}$.
Diversos estudos demonstram que os doentes oncológicos da cabeça e pescoço podem apresentar: dor persistente ${ }^{27}$, dispneia, disfagia e odinofagia ${ }^{27}$, xerostomia, voz alterada ou perda total da voz, perda de peso, cansaço constante, rigidez dos tecidos ${ }^{28}$, traqueostomia e sondas ou tubagem para alimentação, além de diminuição acentuada ou perda da capacidade laborativa. As funções comprometidas (fonação, mastigação, deglutição, respiração e alterações estéticas) relacionam-se claramente com a anátomo-fisiologia de cabeça e pescoço. Frequentemente a cirurgia afecta a fonação, a deglutição e a estética, enquanto a radioterapia afecta predominantemente a deglutição e o gosto ${ }^{1,27-30}$.

Pelo exposto, o tumor maligno da cabeça e pescoço é uma doença mutilante, introduzindo alterações físicas, funcionais, emocionais e sociais importantes, com impacto negativo significativo na Qualidade de Vida do doente $\mathrm{e}^{1,28-30}$

Neste estudo analisa-se resultados da QdVRS de doentes oncológicos incluídos no Projecto de Implementação da avaliação da QdVRS dos Serviços de Otorrinolaringologia e Cabeça \& Pescoço do Instituto Português de Oncologia do Porto (IPOPFG) e simultaneamente procura-se enquadrar esta avaliação no contexto clínico e epidemiológico.

\section{Método}

Participaram neste estudo 380 doentes oncológicos de cabeça e pescoço dos Serviços de Otorrinolaringologia e de Cabeça \& Pescoço do Instituto Português de Oncologia Francisco Gentil do Porto (IPOPFG) que vieram à consulta, incluídos no protocolo de avaliação entre 1- 6 meses após tratamento.

Utilizou-se a versão portuguesa publicada e validada do European Organization for Research and Treatment of Cancer Quality of Life Questionnaire-Core 30 (EORTC QLQ-C30. O QLQ-C30 (versão 3) incorpora 5 escalas funcionais (física, desempenho, cognitiva, emocional e social), 3 escalas de sintomas (fadiga, dor, náusea e vômito), o 
status global de saúde/escala QdVRS e 6 itens simples para avaliação de sintomas ou problemas adicionais (dispneia, perda de apetite, insônia, dificuldades financeiras, obstipação e diarreia) ${ }^{25,26}$. À excepção das escalas funcionais e Status global de saúde/ escala QdVRS, em todas as outras escalas e itens simples, um score elevado indica pior QdVRS. As possibilidades de resposta às perguntas 1 a 28 variam entre 1 - 4 (1 - "Não"; 2 - "Um pouco"; 3 - "Bastante”; 4 - "Muito”). As possibilidades de resposta às perguntas de 29 e 30 variam entre 1 e 7 , em uma escala em que 1 significa "péssima" e 7 significa "ótima”. Os dados obtidos correspondem ao estado do doente durante a última semana. Os dados obtidos foram cotados segundo o Manual da EORTC ${ }^{25,26}$. O testes de hipóteses (teste de Mann-Whitney e teste de Kruskal Wallis) foram usados para verificar a distribuição da população para as variáveis em estudo.

Uma plataforma informática (QoLIP) foi desenhada especificamente para este estudo, sendo composta por unidades de registro obrigatório de variáveis sociodemográficas e clínicas e uma unidade de avaliação de QdVRS passíveis de tratamento estatístico capaz de relacionar a epidemiologia da patologia oncológica de cabeça e pescoço com os scores de QdVRS. Os doentes responderam aos questionários de QdVRS num monitor touch-screen no Gabinete de Qualidade de Vida em Oncologia do IPOPFG, EPE entre Janeiro e Junho de 2010. Apenas alguns doentes necessitaram que lhes fossem lidas as questões devido à baixa literacia.

As variáveis sociodemográficas, hábitos comportamentais (consumo de tabaco e álcool) e clínicas, foram obtidas e transcritas por profissionais de saúde a partir dos processos clínicos e de entrevistas com o doente.
O estudo teve a aprovação da Comissão Ética do Instituto Português de Oncologia do Porto. Todos os doentes concordaram em participar no estudo, deram o consentimento informado e completaram o questionário imediatamente antes da consulta como parte da avaliação de rotina.

\section{Resultados}

\section{Idade e Gênero}

Pela análise da Tabela 1 é possível verificar que as idades da população variam entre 16 e 93 anos, 50,5\% situam-se no grupo etário dos 41-60 anos e 42,2\% colocam-se no grupo etário acima dos 60 anos. O gênero masculino apresenta percentagem muito superior em todos os grupos etários considerados.

\section{Hábitos tabágicos e etílicos}

Como está ilustrado na Figura 1, 50\% dos doentes oncológicos de cabeça e pescoço avaliados são ex-fumantes e verifica-se que os hábitos tabagísticos foram abandonados majoritariamente durante nos últimos 5 anos. Os hábitos tabagísticos e etílicos da população estudada estão evidenciados na Figura $1 \mathrm{a} / \mathrm{b}$.

Verifica-se que a laringe é a localização tumoral mais frequente na população estudada (25\%), seguindo-se a cavidade oral (16\%). O carcinoma espinocelular é o diagnóstico anátomo-patológico mais encontrado (65\%). O estadiamento pela classificação TNM pode ser analisado na figura 2 .

Nesta população verifica-se que, no que concerne à forma de alimentação do doente oncológico de cabeça e pescoço, $80 \%$ têm

Tabela 1 - Características sociodemográficas (Idade e Gênero).

Table 1 - Socio-demographic variables (Age and Gender).

\begin{tabular}{cccccc}
\hline \multicolumn{5}{c}{ Idade / Género (\%) } \\
\hline \multicolumn{2}{c}{ Até 40 Anos (\%) } & \multicolumn{2}{c}{$41-60$ Anos (\%) } & \multicolumn{2}{c}{$>$ 60 Anos (\%) } \\
7.3 (16-40) & \multicolumn{2}{c}{$50.5(41-60)$} & $42.2(61-93)$ \\
\hline Homens & Mulheres & Homens & Mulheres & Homens & Mulheres \\
\hline 90.9 & 9.1 & 78.3 & 21.7 & 84.3 & 15.7 \\
\hline
\end{tabular}




\section{Hábitos tabagísticos}

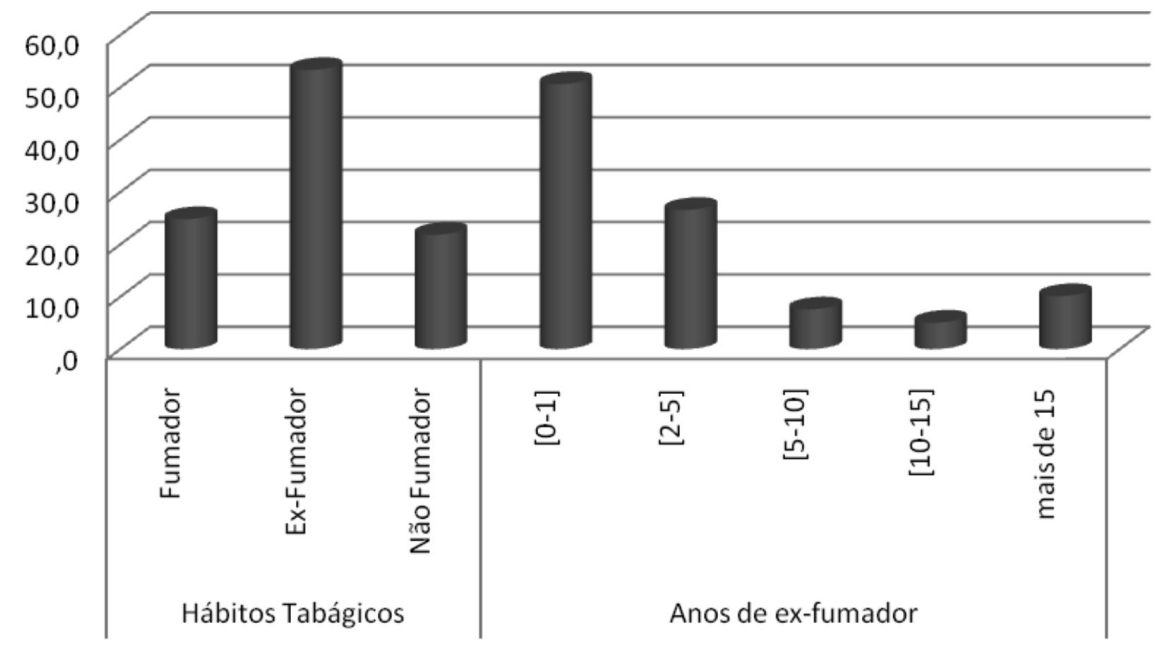

Hábitos etílicos =Vinho = - Cerveja = Bebidas brancas

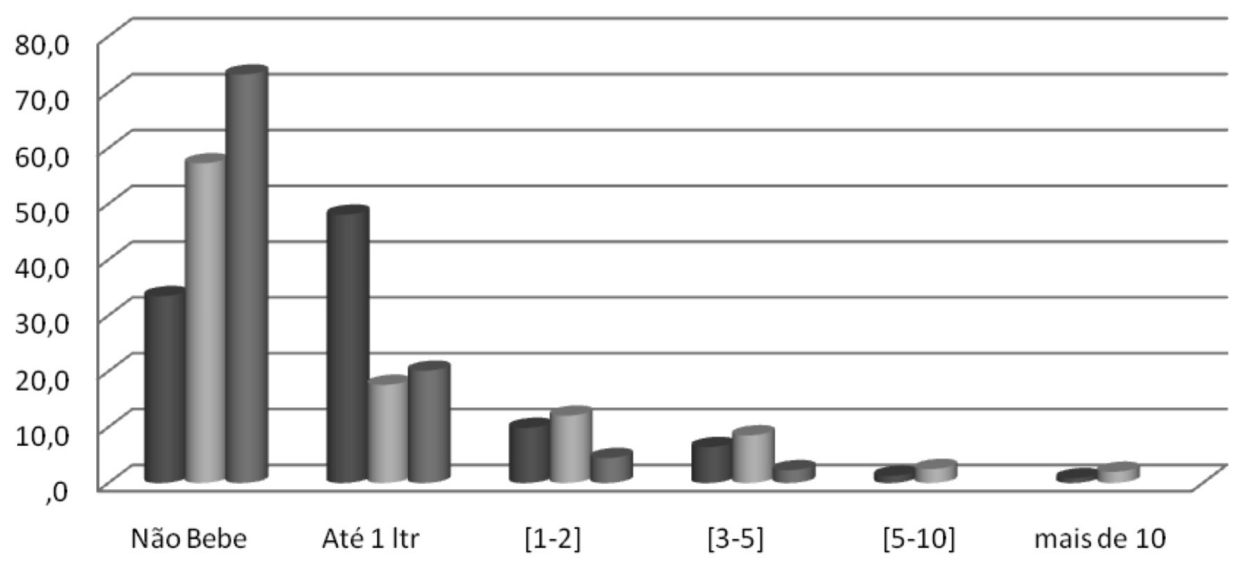

Figura 1 a/b - Hábitos tabagísticos e etílicos.

Figure $1 \mathbf{a} / \mathbf{b}$-Smoking and Drinking habits.

uma alimentação por via oral (Per Os), enquanto os restantes $20 \%$ se distribuem majoritariamente pela percutaneous endoscopic gastrostomy (PEG) e residualmente pela nasogastric intubation (SNG). Ao avaliar a presença de traqueostomia, verifica-se que $70 \%$ nos doentes não apresenta traqueostomia.

\section{QdVRS em função de variáveis} sociodemográficas e clínicas

O teste de Mann-Whitney indica a base da língua como a localização de maior impacto negativo nas mulheres, ao comparar com todas as outras localizações em todas as dimensões consideradas. O teste de KruskalWallis com 95\% de confiança bicaudal permite verificar neste grupo a existência de diferença estaticamente significativa na escala física funcional ( $p$-value de 0,004 ). A diferença de 10 pontos indica diferença clinicamente significativa, podendo se verificar que esta diferença se estende no grupo das mulheres com patologia oncológica da cavidade oral a praticamente todos os domínios, com exceção das escalas funcionais Cognitiva e Social bem como no item simples Dificuldades Financeiras. Salienta-se 


\section{Estadiamento (TNM)}

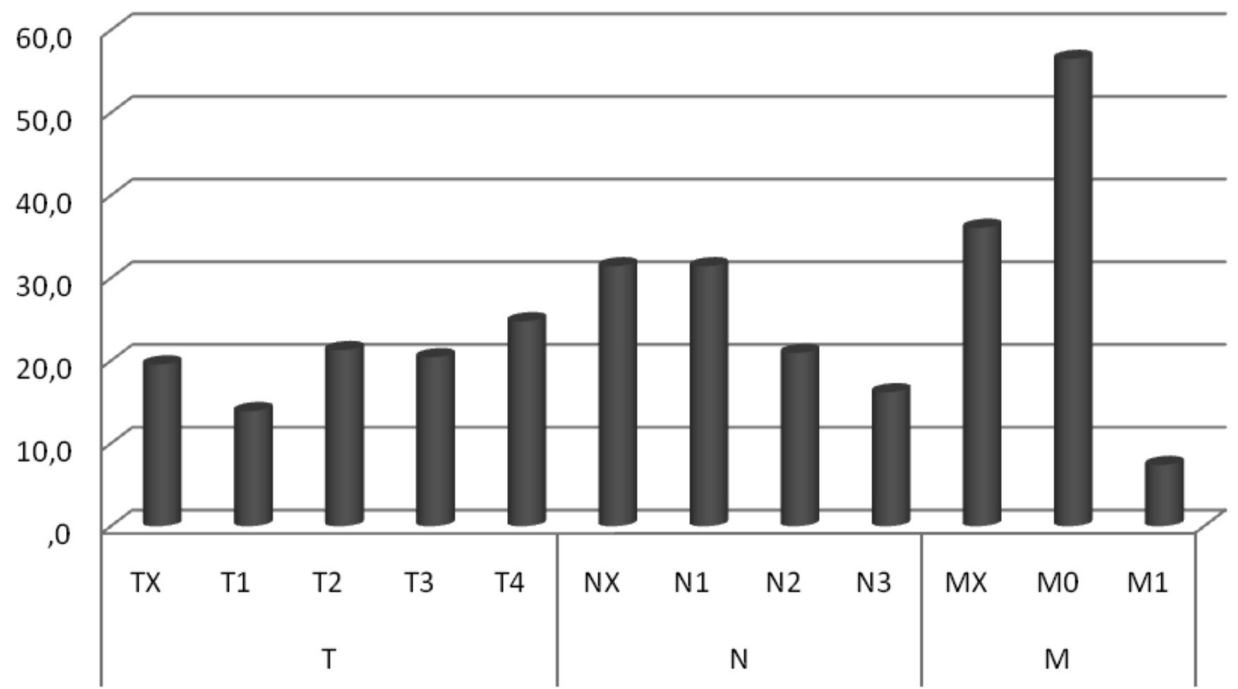

Figura 2 - Estadiamento tumoral.

Figure 2 - Tumor staging.

ainda o grupo das mulheres com patologia oncológica da laringe que reconhecem elevado impacto negativo no Global Health Status. Não se encontra diferença estatística ou clínica nos grupos restantes. Foram considerados os scores de QdVRS em função do estadiamento, constatando-se que o tamanho do tumor ( $\mathrm{T}$ ) apresenta diferença estatisticamente significativa nas dimensões Física ( $p$-value: 0,03$)$ e Insônia ( $p$-value: $0,03)$, com maior impacto negativo em T3 e T4. A metastização regional (N) apresenta diferença estatisticamente significativa na dimensão Dor ( $p$-value: 0,04), com valores mais negativos em N3. Os dados não permitem identificar estas diferenças na análise da Metastização Sistémica (M). Ao considerar a presença de traqueostomia, o teste de Mann-Whitney com 95\% de confiança bicaudal não permite afirmar a existência de diferença estatiscamente significativa nas dimensões consideradas, exceto no item simples Problemas Financeiros, com um $p$-value de 0.005. De igual forma não se verificam diferenças clinicamente significativas. No que concerne à forma de alimentação por PEG ou por SNG, verifica-se que não há diferenças estatisticamente significativas nas escalas e itens considerados. Contudo, há diferenças clinicamente significativas nas escalas funcionais: Física e Desempenho e itens simples: Insônia, Perda de Apetite e Dificuldades Financeiras; e nas escalas de Sintomas: Fadiga; com maior impacto negativo nos doentes portadores de SNG; já na escala funcional Cognitiva, na escala de sintomas a Dor e o item simples Dispneia assinalam maior impacto negativo nos doentes portadores de PEG (Tabela 3).

Ao considerar a presença de traqueostomia, o teste de Mann-Whitney, com 95\% de confiança bicaudal, não permite afirmar a existência de diferença estatiscamente significativa nas dimensões consideradas, excepto no item simples: problemas financeiros com um p-value de 0,005 . No que concerne à forma de alimentação por PEG ou por SNG, verifica-se que não há diferenças estatisticamente significativas nas escalas e itens considerados.

Foram considerados os scores de QdVRS em função do estadiamento, constatando-se que o tamanho do tumor ( $\mathrm{T}$ ) apresenta diferença estatisticamente significativa nas dimensões: Física ( $p$ - value: 0,03 ) e Insônia ( $p$-value: 0,03 ), com maior impacto negativo 
Tabela 2 - Scores de QdVRS em função das variáveis: gênero e localização do tumor.

Table 2 - Quality of Life Scores considering gender and tumor location.

\begin{tabular}{|c|c|c|c|c|c|c|c|c|c|}
\hline \multirow{2}{*}{$\begin{array}{l}\text { EORTC } \\
\text { QLQ C30 }\end{array}$} & & \multicolumn{2}{|c|}{ Laringe } & \multicolumn{2}{|c|}{ Cavidade oral } & \multicolumn{2}{|c|}{ Faringe } & \multicolumn{2}{|c|}{ Base de língua } \\
\hline & & $\begin{array}{c}\text { Homem } \\
n=72\end{array}$ & $\begin{array}{l}\text { Mulher } \\
\mathrm{n}=5\end{array}$ & $\begin{array}{c}\text { Homem } \\
n=43\end{array}$ & $\begin{array}{c}\text { Mulher } \\
\mathrm{n}=15\end{array}$ & $\begin{array}{c}\text { Homem } \\
n=34\end{array}$ & $\begin{array}{c}\text { Mulher } \\
\mathrm{n}=5\end{array}$ & $\begin{array}{c}\text { Homem } \\
\mathrm{N}=29\end{array}$ & $\begin{array}{c}\text { Mulher } \\
\mathrm{n}=6\end{array}$ \\
\hline \multirow{5}{*}{$\begin{array}{l}\text { Escalas } \\
\text { Funcionais }\end{array}$} & Física & 79 & 84 & 76 & 76 & 80 & 80 & 78 & 69 \\
\hline & Desempenho & 80 & 87 & 78 & 79 & 84 & 80 & 77 & 53 \\
\hline & Cognitiva & 86 & 90 & 83 & 89 & 81 & 87 & 83 & 83 \\
\hline & Emocional & 75 & 77 & 77 & 72 & 75 & 63 & 82 & 54 \\
\hline & Social & 86 & 87 & 86 & 81 & 82 & 73 & 84 & 84 \\
\hline \multirow[t]{6}{*}{ Itens Simples } & Dispneia & 16 & 13 & 18 & 4 & 22 & 6 & 14 & 17 \\
\hline & Insônia & 24 & 13 & 29 & 27 & 24 & 6 & 31 & 45 \\
\hline & Perda de apetite & 16 & 20 & 17 & 13 & 19 & 20 & 21 & 22 \\
\hline & Obstipação & 17 & 13 & 15 & 20 & 20 & 6 & 13 & 28 \\
\hline & Diarreia & 4 & 6 & 8 & 6 & 7 & 0 & 3 & 11 \\
\hline & $\begin{array}{l}\text { Dificuldades } \\
\text { Financeiras }\end{array}$ & 24 & 20 & 20 & 22 & 24 & 20 & 21 & 11 \\
\hline \multirow{3}{*}{$\begin{array}{l}\text { Escala de } \\
\text { Sintomas }\end{array}$} & Fadiga & 24 & 24 & 25 & 28 & 26 & 15 & 26 & 50 \\
\hline & Náusea e vômito & 5 & 6 & 5 & 1 & 4 & 20 & 4 & 28 \\
\hline & Dor & 19 & 13 & 23 & 23 & 26 & 17 & 30 & 45 \\
\hline \multicolumn{2}{|c|}{ Global Health Status } & 61 & 38 & 55 & 60 & 58 & 60 & 57 & 35 \\
\hline
\end{tabular}

Tabela 3 - Scores QdVRS em função da forma de alimentação e existência de traqueostomia. Table 3 - Quality of Life Scores considering feeding and tracheotomy existence.

\begin{tabular}{|c|c|c|c|c|c|}
\hline \multirow{2}{*}{$\begin{array}{l}\text { EORTC } \\
\text { QLQ C30 }\end{array}$} & & \multicolumn{2}{|c|}{ Forma de Alimentação } & \multicolumn{2}{|c|}{ Traqueostomia } \\
\hline & & PEG & SNG & Sim & Não \\
\hline \multirow{5}{*}{$\begin{array}{l}\text { Escalas } \\
\text { Funcionais }\end{array}$} & Física & 61 & 49 & 73 & 79 \\
\hline & Desempenho & 66 & 53 & 81 & 79 \\
\hline & Cognitiva & 74 & 87 & 82 & 83 \\
\hline & Emocional & 65 & 62 & 76 & 74 \\
\hline & Social & 70 & 67 & 82 & 84 \\
\hline \multirow[t]{6}{*}{ Itens Simples } & Dispneia & 29 & 7 & 19 & 14 \\
\hline & Insônia & 41 & 60 & 26 & 27 \\
\hline & Perda de apetite & 10 & 20 & 16 & 20 \\
\hline & Obstipação & 31 & - & 16 & 14 \\
\hline & Diarreia & 10 & 6 & 7 & 5 \\
\hline & Dificuldades Financeiras & 33 & 47 & 31 & 22 \\
\hline \multirow{3}{*}{$\begin{array}{l}\text { Escala de } \\
\text { Sintomas }\end{array}$} & Fadiga & 41 & 60 & 30 & 25 \\
\hline & Náusea e vômito & 6 & 10 & 4 & 5 \\
\hline & Dor & 38 & 23 & 23 & 25 \\
\hline \multicolumn{2}{|c|}{ Global Health Status } & 50 & 50 & 62 & 59 \\
\hline
\end{tabular}


em T3 e T4. A metastização regional (N) apresenta diferença estatisticamente significativa na dimensão: Dor ( $p$-value: 0,04), com valores mais negativos em N3. Os dados não permitem identificar estas diferenças na análise da Metastização Sistêmica (M.)

\section{Discussão}

O Instituto Português de Oncologia do Porto assume uma posição de vanguarda e apoia, em parceria com a Universidade Fernando Pessoa, o desenvolvimento de um Sistema de Gestão de Conhecimento na avaliação da Qualidade de Vida por rotina dos doentes oncológicos, com vista à Implementação de uma Unidade de Qualidade em Oncologia.

Considerando a natureza inovadora desta tipologia de intervenção, tornou-se necessária a identificação de Fatores Críticos de Sucesso e a definição de prioridades operacionais e formativas com vista a sensibilizar os profissionais de saúde para a análise epidemiológica e a avaliação Qualidade de Vida Relacionada com a Saúde, com fins clínicos, administrativos e investigacionais.

Quanto às variáveis sociodemográficas, os participantes deste estudo, oriundos dos Conselhos do Norte de Portugal, são maioritariamente do sexo masculino, o que se enquadra no levantamento epidemiológico relativo às taxas de incidência de tumores malignos de cabeça e pescoço apesar de se assistir a um aumento do número de mulheres e um decréscimo no número de homens ${ }^{8,25}$.

De igual forma, a idades dos participantes estão de acordo com a distribuição por idades dos tumores malignos de cabeça e pescoço que consta na literatura, assistindo-se a um aumento do número de doentes geriátricos neste grupo de doentes. Estes resultados são concordantes com a tendência global do envelhecimento da população ${ }^{25,31,32}$.

A análise da amostra quanto ao consumo diário de tabaco enquanto fumante revela que esta é constituída majoritariamente por fumantes ou ex-fumantes de grandes quantidades de tabaco, verificando-se que os ex-fumantes abandonaram os hábitos tabagísticos frequentemente após a data de diagnóstico da doença. Esta é a variável comportamental analisada que mais evidencia o papel do tabaco como fator que predispõe a uma redução da QdVRS, sugerindo a literatura que a cessação tabagística melhora os scores de QDVRS. Assim, além de ser o maior factor de risco dos tumores malignos de cabeça e pescoço, contribui para uma diminuição dos scores de QdVRS ${ }^{33-35}$.

Em concordância com a literatura, a localização dos tumores nesta amostra revela que os tumores malignos da laringe representam aproximadamente $40 \%$ de todos os tumores malignos da cabeça e pescoço, seguidos das localizações cavidade oral e faringe ${ }^{36}$.

De igual forma, salienta-se a importância do diagnóstico precoce em oncologia, que se relaciona frequentemente com estadios de melhor prognóstico e com melhores scores de QdVRS. Também a localização do tumor tem impacto sobre a QdVRS, salientando-se neste estudo a importância de considerar os tumores malignos da cavidade oral e evidenciar a base da língua (orofaringe) nas mulheres, com resultados de QdVRS preocupantes em praticamente todos os domínios $^{25,37-39}$. Verificou-se ainda que o uso de PEG se relaciona com melhores resultados na QdVRS, permitindo considerar a importância da avaliação de QdVRS para a triagem de doentes candidatos a uma destas opções ${ }^{40}$.

Ainda é possível verificar que, quando as variáveis do estudo não estão registradas em todos os doentes oncológicos, não é permitido estudá-las e relacioná-las estatística e clinicamente com os scores de QdVRS. Finalmente, a heterogeneidade da amostra é reconhecida como uma limitação deste estudo, uma vez que os doentes oncológicos de cabeça e pescoço manifestam variações da QdVRS ao longo do tempo e em função das variáveis clínicas, das quais importa salientar o tipo de tratamento, o estadiamento e a localização do tumor.

Face ao exposto, evidencia-se a importância de um correto registro clínico e 
epidemiológico e o elevado interesse da avaliação da QdVRS por rotina na prática clínica oncológica. Reconhece-se, ainda, que a heterogeneidade da amostra estudada é uma limitação neste tipo de estudo, tal como é reconhecido na literatura. A segmentação em função das variáveis clínicas, comportamentais e epidemiológicas permitirá uma análise estatística mais complexa na procura de factores que possam ter impacto nos scores de QdVRS ${ }^{41-44}$.

\section{Conclusão}

A melhoria dos Sistemas de Saúde é uma prioridade em todo o mundo, com enfoque principal nas estratégias destinadas à Saúde Pública e ingerências de carácter médico interventivo.

A articulação entre a investigação clínica e a prática médica é hoje uma questão fundamental para o desenvolvimento de Sistemas de Gestão do Conhecimento que contribuem para a monitorização da Qualidade em Saúde, suportando cientificamente, já em muitos casos, a tomada de decisões, promovendo a optimização de recursos e sobretudo contribuindo para a melhoria da Qualidade deVida dos doentes.

Um doente oncológico de cabeça e pescoço sofre modificações pluridimensionais que podem condicionar consideravelmente a sua QdVRS e justificam a integração da avaliação da QdVRS na prática clínica oncológica. Esta incorporação tem tido aceitação difícil e é pouco realizada em Portugal.

Os tumores malignos da cabeça e pescoço envolvem a anatomia e fisiologia crânio-facial e afetam várias funções: visão, audição, equilíbrio, olfacto, gosto, mastigação, deglutição, respiração, fonação, sistema endócrino e imagem corporal. Os efeitos do diagnóstico e dos tratamentos nos doentes oncológicos refletem-se nos "patient reported outcome results", que devem ser tidos em consideração na decisão terapêutica.

É possível verificar que as mulheres com patologia oncológica da cabeça e pescoço apresentam scores de QdVRS globalmente inferiores, sugerindo que os cuidados oncológicos devem incidir em estratégias dirigidas aos problemas identificados, uma vez que muitas vezes não dependem exclusivamente das variáveis clínicas comumente avaliadas no ciclo da doença. Os resultados de QdVRS, quando interpretados à luz das variáveis sociodemográficas e clínicas, permitem uma melhor avaliação da Patologia Oncológica da Cabeça e Pescoço numa perspectiva epidemiológica, no sentido de compreender o processo saúde-doença e perceber o seu impacto na QdVRS.

\section{Conflito de Interesses}

Os autores declaram, para os devidos fins, que não existe nenhum tipo de conflito de interesses.

\section{Referências}

1. Pimentel FL. Qualidade de Vida do Doente Oncológico. Edição: De autor; 2003.

2. Ministério da Saúde. Plano de Acção para a Saúde. Áreas prioritárias; 2004.

3. Cojocariu OM, Huguet F, Lefévre M, Périé S. Prognosis and predictive factors in head and neck cancers. Bull Cancer 2009; 96(4): 369-78.

4. Stewart BW, Kleihues P. (Eds). World Cancer Report. Lyon: IARC Press; 2003.

5. Víctor Wunsch-Filho. The epidemiology of oral and pharynx cancer in Brazil. Oral Oncol 2002; 38(8): 737-46.
6. Attar E, Dey S, Hablas A, Seifeldin IA, Ramadan M, Rozek LS et al. Head and neck cancer in a developing country: A population-based perspective across 8years. Oral Oncol 2010; 46(8): 591-6.

7. Marur S, Forastiere AA. Head and neck cancer: changing epidemiology, diagnosis, and treatment. Mayo Clin Proc 2008; 83(4): 489-501.

8. Curado MP, Hashibe M. Recent changes in the epidemiology of head and neck cancer. Curr Opin Oncol 2009; 21(3): 194-200. 
9. Pinheiro Pinheiro PS, Tyczynski JE, Bray F, Amado J, Matos E, Miranda AC ry al. Cancer in Portugal /Cancro em Portugal. Lyon: IARC Technical Publication No 38; 2002.

10. De Boer MF, McCornick LK, Pryun JF, Ryckman RM, van den Born BW. Physical and psychosocial correlates of head and neck cancer: A review of the literature. Otolaryngol Head Neck Surgery 1999; 120: 427-36.

11. Llewellyn CD, McGurK ME, Weinman J. Head and neck cancer: To what extent can psychological factors explain differences between health- related quality of life and individual quality of life. Br J Oral Maxillofac Surg 2005; $1-7$.

12. Llewellyn CD, McGurk M, Weinman J. Are psycho-social and behavioural factors related to health relatedquality of life in patients with head and neck cancer? A systematic review. Oral Oncology 2005; 41: 440-54.

13. Hassanein KAAM, Musgrove BT e Bradbury E. Functional status of patients with oral cancer and its relation to style of coping, social support and psychological status. Br J Oral Maxillofac Surg 2001; 39: 340-5.

14. Reinertsen, JL. Physicians as leaders in the improvement of healthcare systems. Ann Intern Med 1998; 128: 833-8.

15. Patel NP, Granick MS, Kanakaris NK, Giannoudis PV, Werdin F, Rennekampff HO. Comparison of wound education in medical schools in the United States, United kingdom, and Germany. Eplasty 2008; 8: 61-7.

16. Shimura T, Yoshimura A, Saito T, Aso R. Unique medical education programs at Nippon Medical School. J Nippon Med Sch 2008; 75(4): 196-201.

17. Kozu T. Medical education in Japan. Acad Med 2006; 81: 1069-75.

18. Iavarone F, Ceci D, Di Florio C, Giovannini S, De Santis M, Ialonardi R et al. Quality of life and social support for women with breast cancer: research study. Prof Inferm 2009; 62(3): 143-8

19. Ballinger RS, Fallowfielda LJ. Quality of Life and Patientreported Outcomes in the Older Breast Cancer Patient. Clin Oncol 2008; 21(2): 140-55

20. Dapueto JJ, Servente L, Francolino C, Hahn, EA. Determinants of Quality of Life in Patients with Cancer: A South American Study. Cancer 2005; 103(5): 1072-81.

21. Gordon DB, Pellino TA, Miaskowski C, McNeill JA, Paice JA, Laferriere D et al. A 10-year review of quality improvement monitoring in pain management: recommendations for standardized outcome measures. Pain Manag Nurs 2002; 3(4): 116-30.

22. Matias KS, Lehn CN, Antunes JLF. Qualidade de vida de pacientes com câncer bucal e da orofaringe por meio do questionário UW-QOL. RPG rev pos-grad 2007;14(2): 139-46.
23. Meeuwsen EJ, German P, Melis RJ, Adang EM, Goluke Willemse GA, Krabbe PF et al. Cost- effectiveness of post-diagnosis treatment in dementia coordinated by multidisciplinary memory clinics in comparison to treatment coordinated by general practitioners: an example of a pragmatic trial. J Nutr Health Aging 2009; 13(3): 242-8.

24. Calaminus G, Barr R. Economic evaluation and healthrelated quality of life. Pediatr Blood Cancer 2008; 50(S5): 1112-5.

25. Silveira, A. Qualidade de Vida do Doente Oncológico da Cabeça e Pescoço do Instituto Português de Oncologia do Porto Francisco Gentil [dissertação de mestrado]. Porto: Instituto Ciências Biomédicas Abel SalazarUniversidade do Porto; 2007.

26. Fayers PM, Aaronson NK, Bjordal K, Groenvold M, Curran D, Bottomley A. On behalf of the EORTC Quality o Life Group. EORTC QLQ-C30 Scoring Manual. Guidelines for assessing Quality of Life in EORTC Clinical Trials. 3rd ed. Brussels, EORTC; 2001.

27. Vieira VP, De Biase N, Peccin MS, Atallah AN. The research questions and methodological adequacy of clinical studies of the voice and larynx published in Brazilian and international journals. J Eval Clin Pract 2009; 15(3): 473-7.

28. Campbell B, Marbella A, Layde P. Quality of life and recurrence concern in survivors of head and neck cancer. The Laryngoscope 2000; 110: 895-906.

29. Tschiesner U, Becker S, Cieza A. Health professional perspective on disability in head and neck cancer. Arch Otolaryngol Head Neck Surg 2010; 136(6): 576-83.

30. Tschiesner U, Linseisen E, Baumann S, Siedek V, Stelter $\mathrm{K}$, Berghaus A et al. Assessment of functioning in patients with head and neck cancer according to the International Classification of Functioning, Disability, and Health (ICF): a multicenter study. Laryngoscope 2009; 119(5): 915-23.

31. Italiano A, Ortholan C, Dassonville O, Poissonnet G, Thariat J, Benezery K et al. Head and neck squamous cell carcinoma in patients aged $>$ or $=80$ years: patterns of care and survival. Cancer 2008; 113(11): 160-8.

32. Chin D, Boyle GM, Porceddu S, Theile DR, Parsons PG, Coman WB. Head and neck cancer: past, present and future. Expert Rev Anticancer Ther 2006; 6(7): 1111-8.

33. Maxwell JH, Kumar B, Feng FY, Worden FP, Lee JS, Eisbruch A et al. Tobacco use in human papillomaviruspositive advanced oropharynx cancer patients related to increased risk of distant metastases and tumor recurrence. Clin Cancer Res 2010; 16(4): 1226-35.

34. Hays JT, Croghan IT, Baker CL, Cappelleri JC, Bushmakin AG. Changes in health-related quality of life with smoking cessation treatment. Eur J Public Health 2010: 2-7.

35. Pöschl G, Seitz HK. Alcohol and cancer. Alcohol Alcohol 2004; 39(3): 155-65. 
36. Cripps C, Winquist E, Devries MC, Stys-Norman D, Gilbert R. Epidermal growth factor receptor targeted therapy in stages III and IV head and neck cancer. Curr Oncol 2010; 17(3): 37-48.

37. Lee MT, Gibson S, Hilari K. Gender differences in healthrelated quality of life following total laryngectomy. Int J Lang Commun Disord 2010; 45(3): 287-94.

38. Cengiz M, Ozyar E, Esassolak M, Altun M, Akmansu $\mathrm{M}$, Sen M et al. Assessment of quality of life of nasopharyngeal carcinoma patients with EORTC QLQ-C30 and H\&N-35 modules. Int J Radiat Oncol Biol Phys 2005; 63(5): 1347-53.

39. Lee MT, Gibson S, Hilari K. Gender differences in healthrelated quality of life following total laryngectomy. Int J Lang Commun Disord 2010; 45(3): 287-94.

40. Morton RP, Crowder VL, Mawdsley R, Ong E, Izzard M. Elective gastrostomy, nutritional status and quality of life in advanced head and neck cancer patients receiving chemoradiotherapy. ANZ J Surg 2009; 79(10): 713-8.
41. Ludwick DA, Doucette J. Adopting electronic medical records in primary care: lessons learned from health information systems implementation experience in seven countries. Int J Med Inform 2009; 78(1): 22-31.

42. Kebede S, Abebe Y, Wolde M, Bekele B, Mantopoulos J, Bradley E. Educating leaders in hospital management: a new model in Sub-Saharan Africa. Int J Qual Health Care 2009; $1-5$.

43. Salas E, Almeida SA, Salisbury M, King H, Lazzara EH, Lyons $\mathrm{R}$ et al. What are the critical success factors for team training in health care? Jt Comm J Qual Patient Saf 2009; 35(8): 398-405.

44. Carvalho J. (2008). Factores Críticos de Sucesso na Implementação de Sistemas de Gestão de Conhecimento [dissertação de mestrado]. Porto: Universidade Portucalense Infante D. Henrique; 2008.

Recebido em: 26/08/10

Versão final apresentada em: 15/03/11

Aprovado em: 11/04/11 